\title{
Investigation of the Role of ABC Transporters in Pyrethroids Resistance in the Major Malaria Vector Anopheles coluzzii from Northern Nigeria
}

\author{
Zilkifil Abulhassan*, Binta G. Kurfi, Sulaiman Sadi Ibrahim, Daha Umar Ishaq, \\ Muhammad Mahe Mukthar \\ Department of Biochemistry, Faculty of Basic Medical Sciences, Bayero University, Kano, Nigeria \\ Email: ^Xikprof@gmail.com
}

How to cite this paper: Abulhassan, Z., Kurfi, B.G., Ibrahim, S.S., Ishaq, D.U., Mukthar, M.M. (2019) Investigation of the Role of ABC Transporters in Pyrethroids Resistance in the Major Malaria Vector Anopheles coluzzii from Northern Nigeria. Open Journal of Immunology, 9, 29-35. https://doi.org/10.4236/oji.2019.93003

Received: March 3, 2019

Accepted: September 27, 2019

Published: September 30, 2019

Copyright (c) 2019 by author(s) and Scientific Research Publishing Inc. This work is licensed under the Creative Commons Attribution International License (CC BY 4.0).

http://creativecommons.org/licenses/by/4.0/

\begin{abstract}
Resistance to currently available insecticides in the major malaria vectors like Anopheles coluzzii is seriously reducing the effectiveness of core vector control tools. Overexpression of ATP binding cassette transporters (ABC transporters) has been implicated in insecticides resistance in the major malaria vector Anopheles funestus and An. gambiae. To identify the potential role of $\mathrm{ABC}$ transporters in pyrethroids resistance in this work, we use verapamil-a p-glycoprotein inhibitor in a synergist bioassay with pyrethroids on one An. coluzzii populations from northern Nigeria. Genomic DNA extraction and SINE 200 PCR established that the Anopheles s. 1 . from Auyo, Jigawa State was An. coluzzii. Pre-exposure to verapamil followed by Permethrin results in $0.12 \%$ knockdown after 1 hour exposure and $10.7 \%$ mortality after 24 hours, which was higher than that for permethrin alone, but not statistically significant. This bioassay result reveals that $\mathrm{ABC}$ transporters possibly do not contribute to the resistance.
\end{abstract}

\section{Keywords}

DNA, Bioassay, PCR, Anopheles coluzzii, Insecticides and Malaria

\section{Introduction}

Malaria is an infection caused by a parasite Plasmodium falciparum, and it's transmitted by the bite of female Anopheles mosquito which is the major malaria vector. Due to the lack of an effective vaccine, the fight against malaria relies mostly on chemotherapy and vector control [1]. 
However, resistance to currently available insecticide is seriously reducing the effectiveness of the vector control tools [2]. The trend in insecticides resistance is becoming alarming because after one half-decade in reduction in the burden and death due to malaria the progress achieved has leveled off, with the number of malaria-related deaths similar for the year 2015 and 2016 [3].

Insecticides resistance in malaria mosquitoes is mainly caused by two mechanisms 1) increased detoxification/clearance of the insecticides by overexpression and/or over activity of the major metabolising enzymes (cytochrome P450s, carboxylesterases, glutathione S-transferases, etc.); and 2) reduced sensitivity of the target of the insecticide due to point mutation (s), for example, the knockdown resistance $(k d r)$ mutations conferring resistance to the voltage-gated sodium channel target of pyrethroids and DDT insecticides [4].

$\mathrm{ABC}$ transporters have been implicated in insecticides resistance in the major malaria vectors, exposure to $\mathrm{ABC}$ inhibitor verapamil synergizes the activity of insecticides in some malaria vectors. Over-expression of ABC transporter can protect insect against insecticide by protecting tissues. ABC-transporters are potential targets of inhibitors which could reduce resistance [5]. However, the role of $\mathrm{ABC}$ transporters in pyrethroids resistance in malaria vectors from northern Nigeria region is lacking and it needs to be established.

\section{Materials and Method}

\section{Sampling Site}

The collection was carried out in Hadiyau, Auyo as shown in Figure 1 and its geographical coordinates are $12^{\circ} 21^{\prime} 36^{\prime \prime}$ North $9^{\circ} 59^{\prime} 8^{\prime \prime}$ East. Auyo is a Local Government Area of Jigawa State in Northern Nigeria. Its headquarters are in the town of Auyo. It has an area of $512 \mathrm{~km}^{2}$ and a population of 132,001 at the 2006 census. The postal code of the area is 731 [6]. The primary activity of the rural population is agriculture with mainly irrigated rice fields.

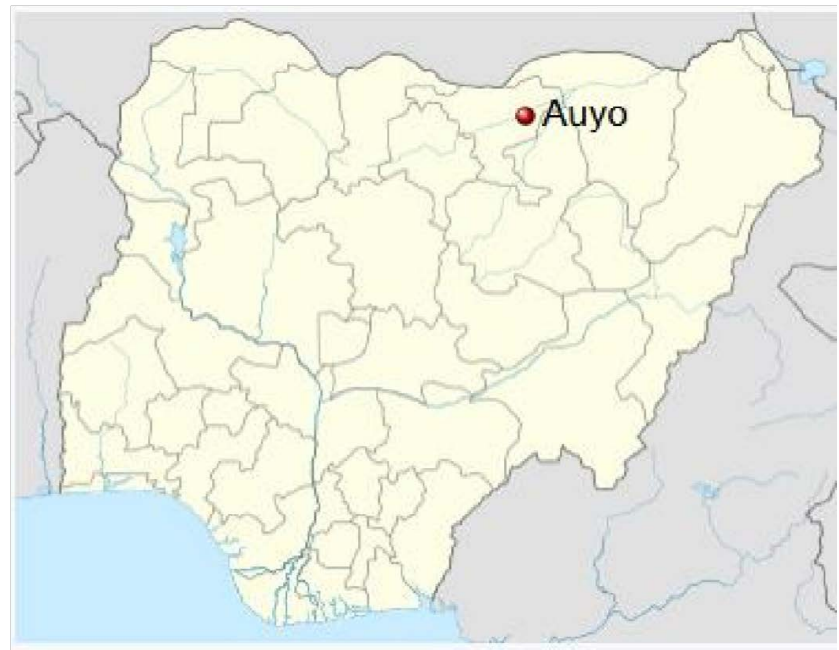

Figure 1. Sampling site Hadiyau Auyo local government, jigawa state. (https://www.en.m.wikipedia.org/ > wiki > Auyo). 


\section{Collection and Rearing of Mosquitoes}

\section{a) Indoor Collection}

Blood-fed female $A$. gambiae s. 1 . adults resting indoor were collected in houses between 04:00 AM to 06:00 AM in Hadiyau, on 14 ${ }^{\text {th }}$ July, 2018 using battery power aspirator and put into the insect rearing cage. The mosquitoes were transported in plastic bug dorm cages and reared in the insectary laboratory at department of Biochemistry, Faculty of Basic Medical Sciences, Bayero University Kano.

\section{b) Mosquito Rearing}

Blood fed females were maintained under standard insectary condition $\left(25^{\circ} \mathrm{C}\right.$ - $28^{\circ} \mathrm{C}$ and $\sim 70 \%$ - $80 \%$ humidity) [7] fed with cotton wool pad soaked with $10 \%$ sucrose solution. In the next few days, these mosquitoes were made to oviposit, after laying eggs, the parent was taken for subsequent experiment and the eggs obtained were put into disposable cups containing water in order to obtain the larvae. The larvae were maintained in a deionized water and fed daily with Tetramin baby fish food. The emerging $\mathrm{F}_{1}$ progenies were used for insect susceptibility bioassays.

c) Morphological Specie Identification

Mosquitoes were identified morphologically as An. gambiae s. l. complex species using the morphological identification keys [8].

\section{Molecular Species Identification}

Genomic DNA was extracted from the parental $\mathrm{F}_{0}$ females using the LIVAK extraction protocol [9].

The species identity of the An. gambiae s. 1 . mosquitoes and the molecular forms of all the An. gambiae s. $s$. were determined according to the SINE PCR method [10].

Species identification primers for $A n$. gambiae Ribosomal DNA (rDNA) intergenic spacer region. 5'-TCG CCT TAG ACC TTG CGT TA-3' SINE 200_F. 5'-CGC TTC AAG AAT TCG AGA TAC-3' SINE 200_R [10].

\section{Insecticide Susceptibility Bioassays}

\section{a) Preparation of Stock Solution}

$0.1 \mathrm{~g}$ of verapamil was dissolved in $10 \mathrm{ml}$ of ethanol and mixed by using a vortex mixer to make $1 \%$ stock solution. $1 \mathrm{ml}$ of the stock solution is added to 4 $\mathrm{ml}$ of ethanol followed by $5 \mathrm{ml}$ of diluent of Dow Corning oil to make a total volume of $10 \mathrm{ml}$. This gives $0.01 \%$ verapamil concentration. A positive control paper was also created by mixing $1 \mathrm{ml}$ of ethanol and $1 \mathrm{ml}$ of Dow Corning oil.

\section{b) Impregnation of Synergist on the Filter paper}

4 Whatman papers $12 \mathrm{~cm} \times 15 \mathrm{~cm}$ were impregnated individually with $1.4 \mathrm{ml}$ of verapamil solution using a pipette dropper, by dropping on the whole surface of the paper individually with the same concentration. The positive control paper was also coated with the mix of oil and ethanol devoid of verapamil.

d) WHO Insecticides Susceptibility Bioassays

Non-blood 2 - 4 days old $\mathrm{F}_{1}$ females were used in WHO susceptibility tests 
[11]. Three replicates of 20 - 25 females were first pre-exposed to verapamil for 1 hour and then transferred to tubes containing $0.75 \%$ permethrin. Insects were exposed to permethrin for $1 \mathrm{hr}$ counting knockdown at $5 \mathrm{~min}, 15 \mathrm{~min}, 30 \mathrm{~min}$, $45 \mathrm{~min}$ and 1 hour. For first positive control, one tube of 20 females was exposed to permethrin alone without pre-exposure to verapamil. For second positive control, 20 females were exposed to $0.01 \%$ verapamil alone without exposure to permethrin. For control 20 females were placed in paper coated with Dow Corning oil plus ethanol with neither permethrin, nor verapamil. Following exposure, mosquitoes were then transferred to a holding tube as shown in Figure 2 and supplied with $10 \%$ sucrose solution and kept overnight under insectary conditions at $25 \%-28^{\circ} \mathrm{C}$ and $\sim 70 \%-80 \%$ humidity [7]. Mortality was recorded for each tube after 24 hours. Data were analysed using Microsoft Excel 2016 and Values are expressed in mean \pm S.D.

\section{Results}

\section{Species and Molecular forms identification}

Seven female An. gambiae s. 1. mosquitoes collected from Auyo were analysed using SINE PCR method for species identification. All of them were An. coluzzii (formerly $\mathrm{M}$ form) as shown in Figure 3 because the PCR product of $479 \mathrm{bp}$ was amplified [10]. Neither An. gambiae s. s. (formerly S form), nor An. arabiensis were detected.

\section{Insecticide susceptibility bioassays}

Adult female An. gambiae mosquitoes from Auyo that were used in conducting insecticide bioassay revealed high resistance to permethrin with only $0 \%$ knockdown after $1 \mathrm{hr}$ exposure (Figure 4 ) and mortality of $8.7 \%$ after 24 hours (Figure 5). However, pre-exposing mosquitoes to verapamil and followed by permethrin resulted in $0.12 \%$ knockdown after $1 \mathrm{hr}$ exposure (Figure 4 ) and $10.7 \%$ mortality after 24 hours (Figure 5), which there is no difference between the groups. Also, mortality in all control groups was consistently $0 \%$ (Figure 5 ). No knockdown was seen when the mosquitoes were exposed to Ethanol + Dow corning oil (Figure 4) proving that the Dow corning oil and the ethanol were not toxic to the mosquitoes and any mortality should be due to presence of insecticide or verapamil Plus insecticide.

\section{Discussion}

From the result, no significant difference was seen between exposure to permethrin alone and pre-exposure to verapamil followed by permethrin. This indicates that $\mathrm{ABC}$ transporter may not be involved in the pyrethroid resistance in populations from Hadiyau, Auyo Northern Nigeria. In contrast, Joseph et al. 2016 [12] reported in $A n$. gambiae s. 1 . collected from rice irrigation fields of Okyereko in Ghana, that An. coluzzii and An. gambiae s. s. expressed resistance to pyrethroids with less than $35 \%$ mortality. However, there is significant increase in mortality to permethrin after a pre-exposure to verapamil. 


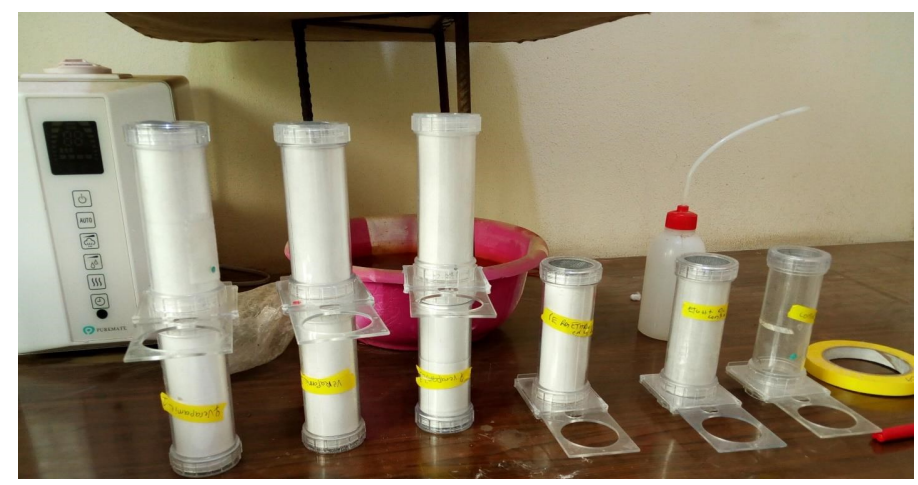

Figure 2. WHO bioassay. 1, 2 \& 3: pre-exposure to verapamil treated papers and then $0.75 \%$ permethrin; 4 : Permethrin treated papers alone; 5: a control paper with ethanol + Dow Corning oil; 6: 6 control of untreated paper).

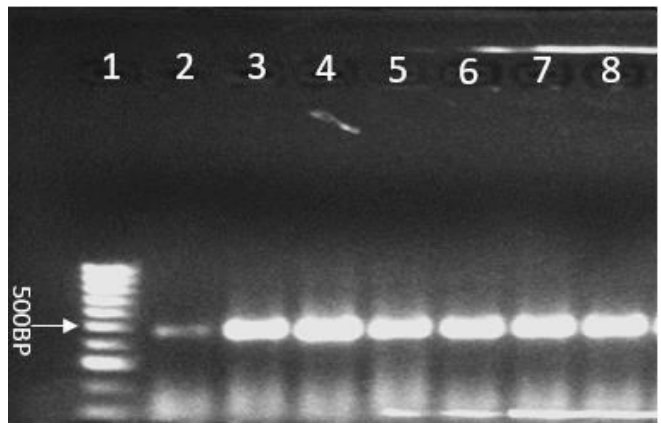

Figure 3. PCR species identification for An. coluzzii from Hadiyau, Auyo. Lane 1 is the DNA standard hyperladder I, lane $2,3,4,5,6,7 \& 8$ are the pcr product of individual mosquito genomic DNA which correspond with the 479 bp characteristic of An. coluzzii.

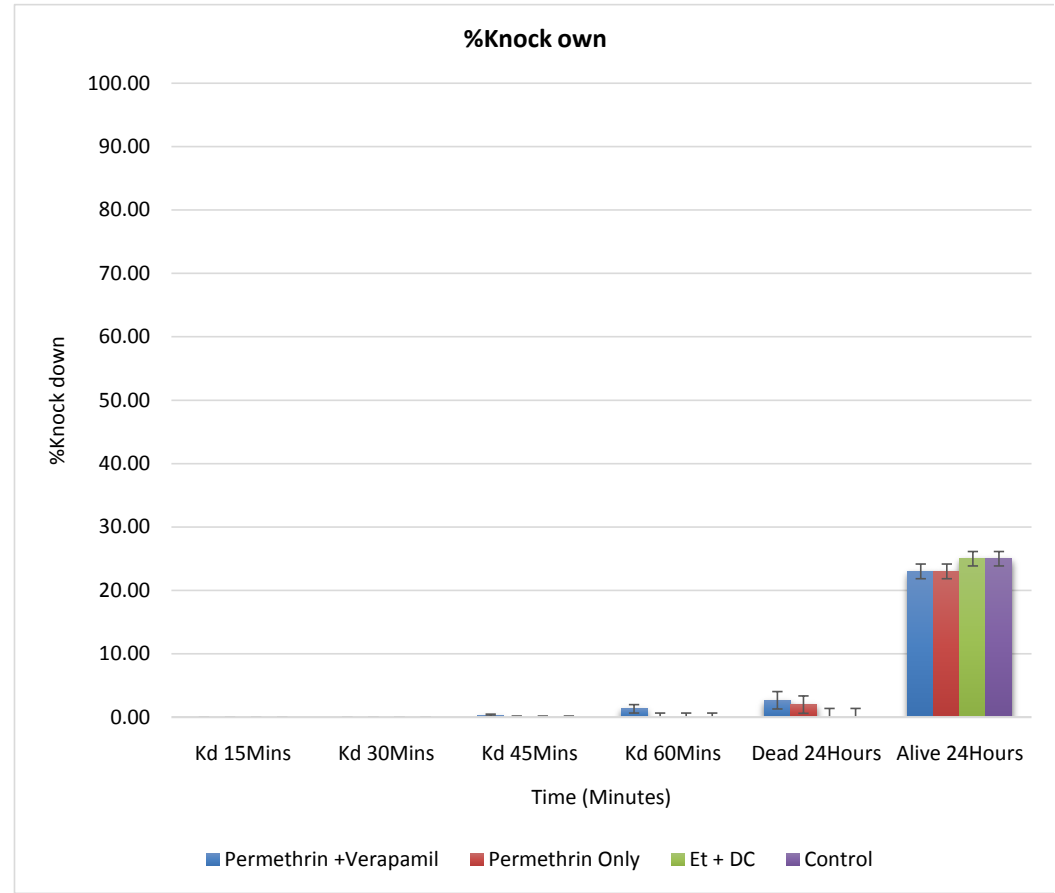

Figure 4. A graph of \% knock down against Time of exposure to insecticide alone and insecticide combined with a synergist. 


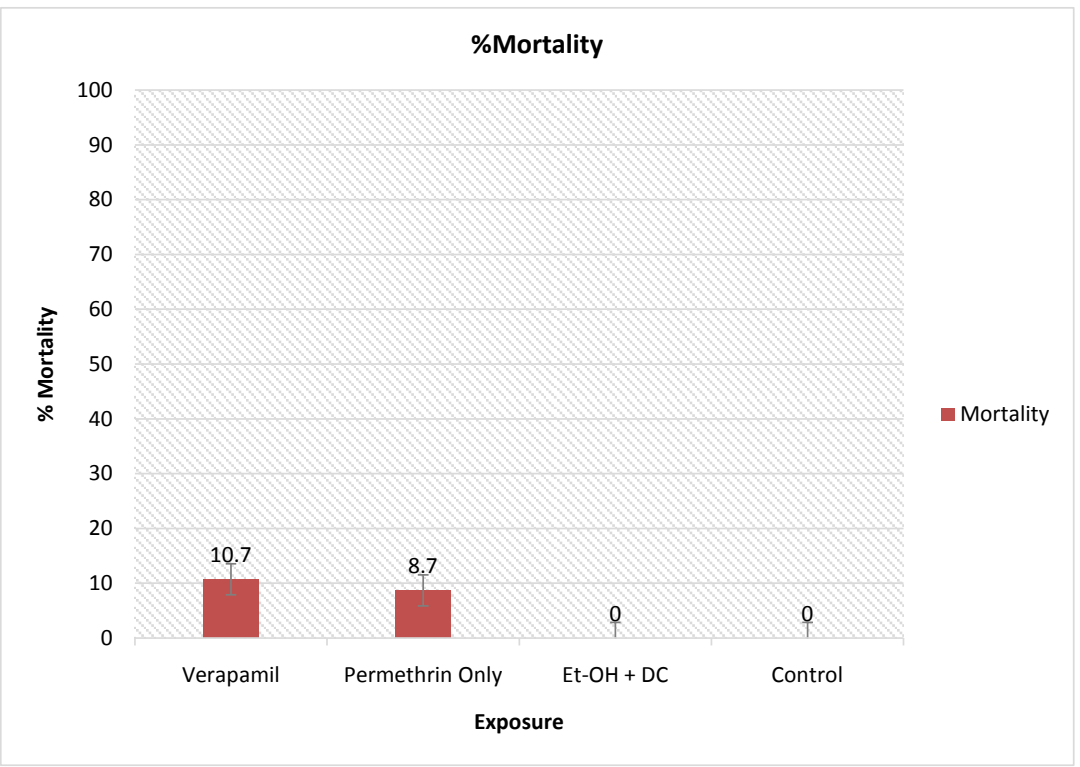

Figure 5. A graph of \% mortality against exposure to insecticide alone and insecticide combined with a synergist.

Also, the permethrin resistance observed were similar to the extremely high resistance reported by Chouaibou et al. 2012 [13], in An. gambiae s. l. collected from rice irrigation fields of Tiassalé in Côte d'Ivoire. The results show a significant increase in mortality to deltamethrin after a pre-exposure to verapamil, suggesting a potential role of $\mathrm{ABC}$ transporters in the resistance mechanisms to this pyrethroid. Several studies have demonstrated that insecticides in mosquitoes were partially regulated through the action of P-gps.

\section{Conclusion}

An. coluzii from Hadiyau is highly resistant to pyrethroid insecticide and this research work reveals that $\mathrm{ABC}$ transporters possibly do not contribute to the resistance. However, further research needs to be carried out with a larger number of mosquito populations from different places in northern Nigeria as well as transcriptional analyses to identify if $\mathrm{ABC}$ transporter is over expressed in the An. coluzii from northern Nigeria.

\section{Conflicts of Interest}

The authors declare no conflicts of interest regarding the publication of this paper.

\section{References}

[1] World Health Organization (2016) World Malaria Report 2016. Geneva.

[2] Ibrahim, S.S., Manu, Y.A., Tukur, Z., Irving, H. and Wondji, C.S. (2014) High Frequency of $k d r$ L1014F Is Associated with Pyrethroid Resistance in Anopheles coluzzii in Sudan Savannah of Northern Nigeria. BMC Infectious Diseases, 14, Article No. 441. http://www.biomedcentral.com/1471-2334/14/441 
https://doi.org/10.1186/1471-2334-14-441

[3] World Health Organization (1998) Tests Procedures for Insecticide Resistance Monitoring in Malaria Vectors, Bioefficacy and Persistence of Insecticides on Treated Surfaces. Report of the WHO Informal Consultation, Geneva, 28-30 September 1998, 43.

[4] Hemingway, J. and Ranson, H. (2000) Insecticide Resistance in Insect Vectors of Human Disease. Annual Review of Entomology, 45, 371-391. https://doi.org/10.1146/annurev.ento.45.1.371

[5] Pignatelli, P., Ingham, V.A., Balabanidou, V., Vontas, J., Lycett, G. and Ranson, H. (2018) The Anopheles gambiae ATP-Binding Cassette Transporter Family: Phylogenetic Analysis and Tissue Localization Provide Clues on Function and Role in Insecticide Resistance. Insect Molecular Biology, 27, 110-122.

https://doi.org/10.1111/imb.12351

[6] NIPOST (2009)

https://ipfs.io/ipfs/QmXoypizjW3WknFiJnKLwHCnL72vedxjQkDDP1mXWo6uco/ wiki/Auyo.html

[7] Garver, L., Das, S. and Dimopoulos, G. (2007) Protocol for Mosquito Rearing ( $A$. gambiae). Journal of Visualized Experiments, 5, 221.

[8] Gillies, M. and Coetzee, M. (1987) A Supplement to Anophelinae of Africa South of Sahara Afro-Tropical Region. The South African Institute for Medical Research, 55, 96-110.

[9] Livak, K.J. (1984) Organization and Mapping of a Sequence on the Drosophila Melanogaster X and Y Chromosomes That Is Transcribed during Spermatogenesis. Genetics, 107, 611-634.

[10] Santolamazza, F., Mancini, E., Simard, F., Qi, Y., Tu, Z. and Della, T.A. (2008) Insertion Polymorphisms of SINE200 Retrotransposons within Speciation Islands of Anopheles gambiae Molecular Forms. Malaria Journal, 7, Article No. 163.

[11] World Health Organization (2016) WHO Susceptibility Tests.

[12] Chabi, J., Baidoo, P.K., Datsomor, A.K., Okyere, D., Ablorde, A., Iddrisu, A., Wilson, M.D., Dadzie, S.K., Jamet, H.P. and Diclaro, J.W. (2016) Insecticide Susceptibility of Natural Populations of Anopheles coluzzii and Anopheles gambiae (sensu stricto) from Okyereko Irrigation Site, Ghana, West Africa. Parasites \& Vectors, 9, Article No. 182. https://doi.org/10.1186/s13071-016-1462-0

[13] Chouaibou, M.S., Chabi, J., Bingham, G.V., Knox, T.B., N’Dri, L., Kesse, N.B., Bonfoh, B., et al. (2012) Increase in Susceptibility to Insecticides with Aging of Wild Anopheles gambiae Mosquitoes from Cote d'Ivoire. BMC Infectious Diseases, 12, Article No. 214. https://doi.org/10.1186/1471-2334-12-214 